\title{
Article
}

\section{Participant Feedback in the Evaluation of Novel Stroke Rehabilitation Technologies}

Meadmore, Katie L., Hughes, Ann-Marie, Freeman, Chris T., Benson, Valerie and Burridge, Jane $\mathrm{H}$.

Available at http://clok.uclan.ac.uk/29472/

Meadmore, Katie L., Hughes, Ann-Marie, Freeman, Chris T., Benson, Valerie ORCID: 0000-0002-0351-4563 and Burridge, Jane H. (2014) Participant Feedback in the Evaluation of Novel Stroke Rehabilitation Technologies. Journal of Rehabilitation Robotics . pp. 82-92. ISSN 2308-8354

It is advisable to refer to the publisher's version if you intend to cite from the work. $10.12970 / 2308-8354.2013 .01 .02 .2$

For more information about UCLan's research in this area go to http://www.uclan.ac.uk/researchgroups/ and search for <name of research Group>.

For information about Research generally at UCLan please go to http://www.uclan.ac.uk/research/

All outputs in CLoK are protected by Intellectual Property Rights law, including Copyright law. Copyright, IPR and Moral Rights for the works on this site are retained by the individual authors and/or other copyright owners. Terms and conditions for use of this material are defined in the policies page. 


\title{
Participant Feedback in the Evaluation of Novel Stroke Rehabilitation Technologies
}

\author{
Katie L. Meadmore ${ }^{1, *}$, Ann-Marie Hughes ${ }^{2}$, Chris T. Freeman ${ }^{1}$, Valerie Benson ${ }^{3}$ and \\ Jane H. Burridge ${ }^{2}$
}

${ }^{1}$ Faculty of Physical Sciences and Engineering; ${ }^{2}$ Faculty of Health Sciences; ${ }^{3}$ Faculty of Social and Human Sciences, University of Southampton, Southampton, SO17 1BJ, UK

\begin{abstract}
Purpose: Stroke participant perspectives are used to evaluate a novel rehabilitation system employing electrical stimulation (ES) technology combined with robotic assistance and virtual reality. The broader implications of such feedback for future technological development are discussed.

Method: While supported by a robot, ES was applied to the triceps and anterior deltoid muscles of 5 chronic stroke participants with upper limb impairment to assist them in completing functional, virtual reality tracking tasks. Advanced ES controllers adjusted the amount of ES applied on each attempt to improve accuracy and maximise voluntary effort. The system was evaluated in terms of participants' perspectives, expressed during a semi-structured interview, and clinical outcome measures.

Results: The rehabilitation system was well accepted by participants and viewed positively, despite mixed opinions regarding effectiveness. Feedback demonstrated an alignment in participants' perceptions of reduced impairment and clinical outcomes, in which a significant $(p<0.001)$ mean change of 9.3 in Fugl-Meyer scores was observed. Participant feedback also provided insight into individual differences observed in clinical outcomes. From our findings six key issues regarding effectiveness, muscles trained, system flexibility and portability, possible discomfort and the value of participant perspectives emerged that may be relevant for researchers developing new rehabilitation technologies.
\end{abstract}

Conclusion: Participant feedback via a semi-structured interview provided important insight into the usability and effectiveness of using this system as a platform for upper limb stroke rehabilitation.

Keywords: Participant perspectives, functional electrical stimulation, upper limb, motor recovery.

\section{INTRODUCTION}

Approximately one third of people who suffer a stroke are left severely disabled, dependent on others for activities of daily living (ADL), and require some form of rehabilitation [1, 2]. Upper limb dysfunction is particularly problematic, impacting on many ADL, such as feeding and dressing.

Electrical stimulation (ES) and robotic therapy are among many rehabilitation techniques shown to reduce impairment in the upper limb [3-5]. However, standardised measures of performance, such as the Fugl-Meyer Assessment [6] and the Action Research Arm Test [7], may not capture all the positive and negative aspects associated with a technology [8]. This has led to an increase in the use of patient-centred outcome measures to provide insight into the technology's effect on patients' quality of life $[8,9]$. In addition, patients' perspectives of therapeutic interventions have been used to inform and improve design of technologies, especially novel rehabilitation technologies [8-14]. This includes the usability of the technology, as well as consideration of personal

*Address correspondence to this author at the Electronics and Computer Science, University of Southampton, Southampton, SO17 1BJ, UK;

Tel: 02380 592735; E-mail: klm@ecs.soton.ac.uk rehabilitation goals $[12,13,15]$, which may determine the tasks to be practiced, the skill that patients expect to regain, and affect compliance and confidence in using the technology $[9,12,16]$.

The rehabilitation system reported in this paper is termed SAIL: Stimulation Assistance through Iterative Learning, and employs virtual reality, robotic and ES technologies to help stroke participants recover movement in their upper limb. The system provides a controlled and safe environment for participant's to receive finely-tuned, personalised ES assistance, and comprises a substantial development over an earlier system which established the use of advanced ES controllers in upper-limb stroke rehabilitation [17, 18]. In addition to combining robotic and ES technologies, the principal novelty of this work is the use of Iterative Learning Control (ILC) to mediate the ES applied to the triceps and anterior deltoid of the participant. ILC is a technology transferred from industrial robotics, and uses data recorded over previous attempts (or 'trials') of the task in combination with a biomechanical representation of each participant's arm, to calculate the level of ES required to maximise motor accuracy on a trial by trial basis $[19,20]$. The biomechanical model captures the dynamic behaviour of the arm in response to applied ES by using a set of differential equations 
that relate the applied ES to the resulting arm movement (see [21] for details). In this way, ILC provides enough ES to assist performance while encouraging participants to exert voluntary effort to accurately complete the task, an important factor associated with increased positive therapeutic effects [4].

The main aims of this paper are to (1) evaluate the SAIL system from five stroke participants' perspectives and compare this data to clinical outcome measures and, (2) based on our findings, discuss key issues and ideas that researchers may find useful to take into account when developing new rehabilitation technologies. To do this our objectives were 1) to evaluate the feasibility of using SAIL for upper limb rehabilitation with stroke participants by comparing clinical outcome measures pre and post 18 sessions using SAIL; 2) to gain understanding of participants experience in using the SAIL rehabilitation system, in terms of effectiveness and usability; 3 ) to gain insight into how the SAIL system could be improved in the future; 4) and to gain insight into how perceived outcomes relate to more objective measures of performance. To be clear, in line with previous work [18] we anticipated an improvement in pre to post clinical outcome measures but how this would relate to participants perceptions of improvement was unclear.

\section{METHOD}

Full engineering design $[19,20]$ and clinical results of the feasibility trial [22] have previously been reported and are therefore only briefly described here.

\section{A. SAIL Feasibility Trial}

Following ethical approval and informed consent, five participants with chronic stroke were recruited to this feasibility study. Participant characteristics are reported in Table $\mathbf{1}$. Inclusion criteria were: i) participants aged $30-75$ years; ii) ES produced movement without undue discomfort; iii) participants could comply with study protocol; iv) participants could communicate effectively; v) participants could give informed consent; vi) participants had suffered a stroke causing hemiplegia for at least 6 months and vii) impaired upper limb that included an inability to effectively extend the elbow in reaching. Exclusion criteria were: i) any active device implant; ii) any metal implant in upper limb; iii) uncontrolled epilepsy; iv) pregnancy; v) any serious or unstable medical or psychological condition or cognitive impairment that would compromise the participants safety or successful participation in this study (this includes any additional upper limb joint problems); vi) interpreter required; vii) participation in another upper limb physical rehabilitation study.

Participants attended 18, 1 hour training sessions over a 6-8 week period (consisting 2-3 sessions per week). During training, the participants' impaired arm was supported against gravity by a robotic support and ES was applied to their triceps and anterior deltoid to help practice 3D reaching tasks (Figure 1). The participants task was to track a slowly moving ball with their impaired arm along a specified trajectory that was displayed in a virtual reality environment on a computer screen in front of them (Figure 1).

At the beginning of each session, the therapist placed electrodes over the muscle body of the anterior deltoid and triceps of the impaired arm. Participants were then seated at the workstation and the participant's hemiplegic arm was loosely strapped to the upper limb support mechanism. The participants viewed a screen (which was located in front of them and to the hemiplegic side) that showed a virtual reality environment displaying the trajectory to be tracked and a representation of the participant's arm (that mirrored the participant's movements in real-time; see Figure 1).

Table 1: Participant Demographics

\begin{tabular}{|c|c|c|c|c|c|c|}
\hline Participant & Age & Gender & $\begin{array}{c}\text { Time since stroke } \\
\text { (months) }\end{array}$ & Side of Lesion & $\begin{array}{c}\text { Type of } \\
\text { stroke }\end{array}$ & $\begin{array}{c}\text { Handedness prior } \\
\text { to stroke }\end{array}$ \\
\hline \hline 1 & 58 & F & 11 & right & infarct & right \\
\hline 2 & 33 & M & 49 & left & rinfarct & right \\
\hline 3 & 40 & M & 52 & right & infarct & right \\
\hline 4 & 67 & M & 77 & right & infarct & right \\
\hline 5 & 65 & F & 13 & & \\
\hline
\end{tabular}




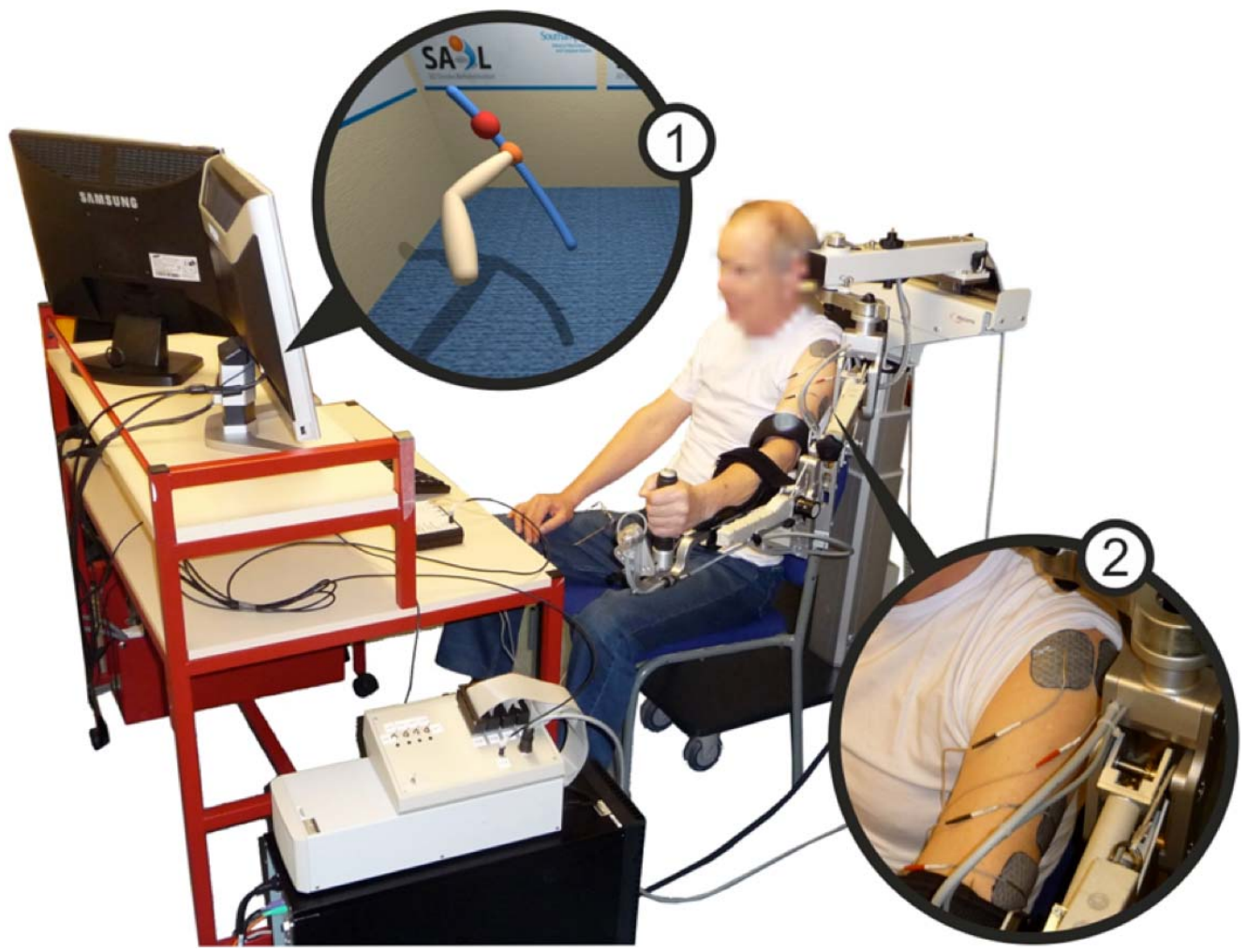

Figure 1: The SAIL system set-up. Bubble 1 shows the virtual reality tracking task with real-time image of the participant's arm as they progress through the trial. Bubble 2 shows ES electrode pads placed on the triceps and anterior deltoid.

The frequency of stimulation was fixed at $40 \mathrm{~Hz}$ in all tests, with a pulse width controlled in real-time by the ILC algorithms. At the beginning of each session, upper limit stimulation amplitude (determined by the participant) was fixed to ensure participant comfort and safety. A workspace in which participants could extend to their full range of movement with assistance from ES was also established, by calculating the spatial coordinates from the highest point that the participant could reach with their impaired arm when ES was applied to both muscle groups, the lowest point closest to the participant's ipsilateral thigh, and a front point relating to elbow extension directly in front of the participant. There were 9 possible trajectories that could be tracked; each could be in one of three orientations relating to space in front and to the hemiplegic side (centre, off-centre and far) and one of three lengths (proximal, middle, and distal). In addition, the ball could move along the trajectory at one of two speeds (5 or 10 seconds), requiring fast or slow tracking movements.

In each task, participants completed 6 trials tracking the same trajectory. A 15 second rest period between iterations was designed to reduce fatigue, and was extended if necessary. Participants started each movement from the same initial position, which was determined at the start of the first trial. Between each trial, the ILC software updated the ES signal applied to each muscle. To do this, the advanced controller uses performance data recorded from the previous trial together with a dynamic model of the arm in order to precisely assist tracking performance during the next attempt $([19,20,21]$ for more details regarding the ILC model parameters). For feedback, participants saw a real-time image of their arm on the computer screen as they tracked the ball and the ball changed colour to indicate performance accuracy.

At the start and end of each session, participants also completed four single unassisted tracking trials (i.e. with no assistance from ES) [22]. Upper limb motor impairment and function were assessed using the FuglMeyer (F-M) [6] and Action Research Arm Test (ARAT)[7], respectively, in two assessments prior to the training sessions (to establish baseline performance) and one assessment a maximum of two days post completion of the training sessions. These clinical outcome measures are valid and reliable measures for use with stroke participants $[6,7,22]$.

\section{B. Interview}

Following completion of the post-assessment session, participants took part in a semi-structured 
Table 2: Question Set Used and Likert Responses

\section{Category/Statement/Question} Question
Style

\begin{tabular}{|c|c|c|c|c|}
\hline \multicolumn{5}{|c|}{ Likert Responses } \\
\hline 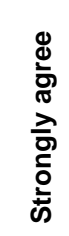 & $\begin{array}{l}\text { ఖ } \\
\text { 훈 }\end{array}$ & 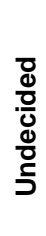 & 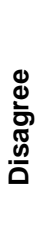 & 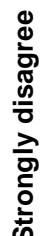 \\
\hline
\end{tabular}

\section{A. System Effectiveness}

\begin{tabular}{|c|c|c|c|c|c|c|}
\hline 1. I am now more aware of my affected arm & Likert & 1 & 3 & 1 & & \\
\hline 2. My arm feels weaker & Likert & & & & 4 & 1 \\
\hline 3. My arm feels tighter & Likert & & 2 & & 3 & \\
\hline 4. I can reach out with my arm more easily & Likert & 1 & 2 & & 2 & \\
\hline 5. I can now pick up objects & Likert & 1 & & 1 & 2 & 1 \\
\hline 6. Are you now able to do things that you could not do before? & Open & \multicolumn{5}{|c|}{$\begin{array}{l}\text { Yes/No. Please give examples and provide as } \\
\text { much information as possible }\end{array}$} \\
\hline 7. Are you now able to do things better than you could before? & Open & \multicolumn{5}{|c|}{$\begin{array}{l}\text { Yes/No. Please give examples and provide as } \\
\text { much information as possible }\end{array}$} \\
\hline 8. Can you now perform any two handed tasks more easily? & Open & \multicolumn{5}{|c|}{$\begin{array}{l}\text { Yes/No. Please give examples and provide as } \\
\text { much information as possible }\end{array}$} \\
\hline $\begin{array}{l}\text { f caregiver/spouse/relative is also at the session ask them: } \\
\text { i. Do you think that (participant name) is now able to do things } \\
\text { that he/she could not do before? } \\
\text { ii. Do you think that (participant name) is now able to do things } \\
\text { better than he/she could before? }\end{array}$ & Open & \multicolumn{5}{|c|}{$\begin{array}{l}\text { Yes/No. Please give examples and provide as } \\
\text { much information as possible }\end{array}$} \\
\hline \multicolumn{7}{|l|}{ i. System Usability } \\
\hline 9. I did not find the treatment enjoyable & Likert & & & & 4 & 1 \\
\hline 10. It was easy to understand what I had to do & Likert & 2 & 3 & & & \\
\hline 11. It was difficult to put my arm in the arm holder & Likert & & & & 4 & 1 \\
\hline 12. The arm holder was comfortable & Likert & & 4 & & 1 & \\
\hline 13. The stimulation was uncomfortable & Likert & & & & 5 & \\
\hline 14. The target (i.e., the moving ball) was easy to see & Likert & 1 & 4 & & & \\
\hline 15. The trajectory (i.e., length, height, direction) was easy to see & Likert & 1 & 4 & & & \\
\hline 16. I did not understand the graphs showing my performance & Likert & & 1 & & 4 & \\
\hline
\end{tabular}

\section{Questions about how the system could be improved}

17. Adding games would add to my motivation and enjoyment of the treatment

18. I would not like to have more arm muscles stimulated

19. How do you think the task could be improved?

\begin{tabular}{|c|c|c|}
\hline Likert & & \\
\hline Likert & & \\
\hline Open & & \\
\hline
\end{tabular}

\begin{tabular}{|l|l|l|l|}
\hline 3 & & 2 & \\
\hline & & 4 & 1 \\
\hline
\end{tabular}

\section{General Questions}

20. I would not recommend the treatment to other people who have had a stroke

21. I would have liked to have continued longer with the treatment

22. Looking back on it, was taking part in this study worthwhile for you?

\begin{tabular}{|c|c|c} 
& Likert & \\
\hline & Likert & 1 \\
\hline & Open & Yes/No; \\
\hline & Open & \\
\hline
\end{tabular}

23. What were the worst aspects of it?

24. What were the best aspects of it?

Open

25. If we could design the ideal rehabilitation system describe five features it should have:

26. If we could stimulate more muscles which movements would you like?

\begin{tabular}{|c|c|}
\hline Open & \\
\hline Open & \\
\hline
\end{tabular}

Note that responses corresponding to the open ended questions are presented in the results section. 
interview. This comprised of 26 open-ended and closed questions corresponding to: effectiveness; usability; improvement and general aspects of the SAIL system and research study (Table 2). Closed questions required a response regarding how much the participant agreed or disagreed with a given statement on a 5 point Likert scale, from Strongly Disagree to Strongly Agree. Likert answers were comprised of both positive and negative statements. Open-ended questions were completely open or asked yes/no questions that then required open-ended qualifications to gain greater insight about that aspect of the system. The interviewer encouraged participants to provide as much additional information as possible.

The question-set was developed in-house by therapists and psychologists working in the field of stoke rehabilitation and rehabilitation technology, and was informed by previous work [18]. The interviews were purposefully designed to be short and easy to follow, and lasted between 30-45 minutes. All interviews took place at the Faculty of Health Sciences and were conducted by a psychologist who was independent to the study to ensure that all participants were as honest and open as possible.

\section{Data Analysis}

\section{Clinical Outcome Measures}

The F-M (motor component only) assesses the degree of motor impairment in the upper limb through examination of how well participants can complete different gross movements with the impaired arm. The ARAT assesses motor function via assessing the number of motor activities the participant can complete (such as lifting different sized blocks on to a shelf and relocating different sized small objects). The maximum score for the F-M was 66 and the maximum score for the ARAT was 57. In line with previous work [18], the data from the two pre-intervention assessment sessions were tested for differences using a t-test and then averaged for baseline performance. A one-tailed, paired t-test, with a significance level of $p<.05$, was used to compare baseline and post-intervention F-M and ARAT outcome measures. A one-tailed test was used as improvements in motor function from pre to post intervention were anticipated.

\section{Interview Data}

The quantitative data provided by the Likert scale items were analysed using descriptive summary statistics. The open-ended questions provided qualitative data that were analysed in a descriptive manner using thematic analysis. Thematic analysis involves familiarisation with data by reading and rereading the responses of all participants until key themes or categories are identified. These themes were then categorised and coded. Quotes were chosen as being the most representative in their group to accurately illustrate the participants' perceptions. There was no saturation of data.

\section{RESULTS}

\section{A. Clinical Outcome Measures}

Adherence was excellent with all 5 participants attending all training and assessment sessions. The results of the F-M and ARAT assessments are presented briefly to enable them to be discussed in the context of the results of the semi-structured interviews (for full clinical results see [22]). As shown in Table $\mathbf{3}$, F-M scores were shown to increase pre- to postintervention, $t(4)=-4.54, p=0.005$, indicating that impairment in the upper limb reduced. No changes were found for the ARAT, $t(4)=-0.34, p=0.37$.

Table 3: Assessment Scores for the ARAT and F-M at Baseline and Post-Training Sessions

\begin{tabular}{|c|c|c|c|c|}
\hline \multirow{2}{*}{ P. Id } & \multicolumn{2}{|c|}{$\operatorname{ARAT}\left(57^{\mathrm{a}}\right)$} & \multicolumn{2}{|c|}{ F-M (Motor; $66^{b}$ ) } \\
\hline & Baseline $^{d}$ & Post-Intervention & Baseline $^{d}$ & Post-Intervention \\
\hline 01 & 0 & 1 & 9.5 & 20 \\
\hline 02 & 7 & 10 & 19 & 33 \\
\hline 03 & 9 & 10 & 31 & 44 \\
\hline 04 & 4 & 0 & 16 & 21 \\
\hline 05 & 12 & 13 & 42 & 46 \\
\hline Mean(SD) & 6.4 (4.62) & 6.8 (5.89) & 23.5 (12.95) & 32.8 (12.28) \\
\hline
\end{tabular}

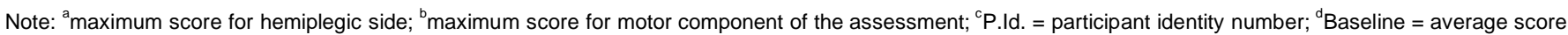
collapsed over the two pre-intervention assessments. 


\section{A. Interview Data}

A summary of the Likert scores can be seen in Table 2.

\section{a) Usability}

Overall, the feedback provided by participants was positive with respect to acceptance of the technology and usability of the system. Participants' reported favourably in terms of comfort and ease of use of the stimulation and robot. All participants found the tracking tasks easy to understand and enjoyable, with one participant commenting: 'Thoroughly enjoyed it - been worthwhile' (Participant 3).

In addition, the immediate feedback provided by the 3D environment and graphical displays helped participants to monitor their progress, within and between trials. Indeed, one participant commented that: 'Seeing the robotic arm on the computer screen and following the green ball with the [aid of] stimulation' (Participant 4), was one of the best aspects of the study. This was important as an understanding of the task, visual cues and feedback regarding progression is all factors that facilitate motivation and willingness to engage in rehabilitation [16].

\section{b) System Effectiveness}

Participants' responses were mixed in terms of the effectiveness of the system. Participants all reported that their arm felt less weak following the end of intervention and that they were more aware of their impaired arm:

Moving the arm in general is better; there is more movement in the arm (Participant 2)

However, only one participant felt that they could now pick up an object more easily. The discrepancy in responses seemed to stem from what the participants perceived the definition of effectiveness to be, with one participant and one carer distinguishing between the fact that although the impaired arm was more mobile this did not transfer to functional changes:

Although arm is more mobile, [I] still cannot do the things that I would like to -there is no functional improvement; for example, I cannot pick up cup of tea with my hand. (Participant 3)

Although [participant] shows more movement, she is still not able to do normal things such as opening a jar, buttering toast etc. at home. (Spouse of Participant 1)
Two participants reported that their arm felt tighter following the intervention, and that they could not reach out more easily with their impaired arm. Interestingly, these two participants also showed the smallest changes on the F-M. This demonstrates an alignment in participant awareness and clinical outcome measures. The other three participants did not think that their arm felt more tight and felt that they could reach out more easily with their impaired arm.

\section{c) Improvements to the System}

All participants would have liked more muscles to have been stimulated. The most popular movements to be assisted by ES involved the hand, wrist and fingers and shoulder.

\section{More shoulder movements (Participant 1) \\ Wrist, fingers and arm raising (Participant 4) \\ Treatment for hand as well as arm (Participant 5)}

Although two participants were keen to use ES for all of their weak muscles.

All of those that are weak as a result of the stroke (Participant 3)

\section{Arm, leg and fingers (Participant 2)}

Participants were mixed about whether adding games, rather than having to track the ball, would add to the motivation and enjoyment of the treatment $(3 / 5$ felt that it would and $2 / 5$ felt the tasks were fine as they were), highlighting individual differences in rehabilitation needs and desires.

[I] think the task is pretty much what it needs to be (Participant 3)

I would like more variety in the tasks (Participant 5)

\section{d) General}

All participants responded favourably that they would recommend the treatment to other people who have had a stroke and that taking part in the study had been worthwhile. However, responses varied concerning duration of the treatment, with only one participant reporting that they would have liked to have continued the treatment for longer. Three participants felt 18 sessions was long enough and 1 participant was undecided.

Responses concerning the best aspects of the study could be divided into 3 categories: Physical 
improvements in the arm (e.g., 'The improvement in the movement in the arm' - Participant 2); Motivational factors associated with physical improvements (e.g., 'Being able to see the improvement in the lines on the computer [graphs] - Participant 2); and a Sense of helping yourself and others (e.g., 'Trying to get arm to work again and volunteering so that people in the future may benefit - Participant 5).

Travel time and temporary physical discomfort were considered the worst aspects of the study. One participant experienced muscle ache of the shoulder and another pain in the hand and wrist. Note that this discomfort was temporary, occurring for a short period immediately following some of the training sessions. In addition, two participants lived over 40 miles away from the University testing centre and the relative of one participant specified that 'Commitment of three times a week is too much' (Relative of Participant 2), despite having felt that the training schedule would not be a problem at the start of the study.

\section{DISCUSSION}

The aim of this paper was to evaluate the SAIL system for its feasibility for providing upper limb rehabilitation to stroke participants in terms of usability and effectiveness. Usability and effectiveness were evaluated by analysing the data from the feedback of five chronic stroke participants who underwent 18 training sessions using SAIL. Effectiveness was also evaluated with traditional objective clinical outcome measures.

Overall the participants provided very favourable views regarding the SAIL rehabilitation system, demonstrating that the technology was well accepted by the participants, despite mixed views regarding the effectiveness of the system. Specifically, they found it easy and comfortable to use and there were no issues or concerns in using the ES to facilitate training. Thus, we feel that the usability and user friendliness of SAIL as an upper limb rehabilitation platform for chronic stroke participants was established.

In terms of effectiveness, the clinical outcome measures of the study provided mixed results. Specifically, in line with previous work [17], the clinical data showed a significant improvement from baseline to post-intervention for F-M scores but not for ARAT scores. While it is disappointing that no significant change was found for the ARAT, this finding is not surprising given that the ARAT contains many functional tasks that require hand function and the SAIL system trained only proximal upper limb joints. One of the study objectives was to compare these clinical outcomes to the participants' own perceptions of improvement. Interestingly and importantly we found consistent findings. In general, participants reported that the arm felt more mobile and that they had a better range of movement; however, some participants commented that despite these general motoric improvements, there was no translation into functional upper limb improvements. This left some participants feeling disappointed with their improvement, despite noticeable reductions in gross motor impairment. Unsurprisingly, participants felt that more functional improvements may have been observed if the system had targeted the hand as well as the triceps and anterior deltoid. Improvement in hand function was an important goal to the participants in this study and they reported that they would like ES to be applied to additional muscles to assist opening the hand for grip and grasp functions.

Participants also commented that they felt that their arm was less weak than before treatment and that they felt more aware of it (that it 'belonged' to their body again). These were both positive aspects of the intervention (both physically and psychosocially) that were not captured when reporting standardised clinical assessments of motor function. This highlights that there may be changes in impairments that are relevant and meaningful to participants that may not be picked up when using standardised assessments. Thus, the participant perspectives and the clinical outcomes have demonstrated significant changes in motor impairment over the course of the intervention, indicating that SAIL may be an effective platform for the rehabilitation of the upper limb post-stroke.

From the participant interviews, we were also able to establish that the two participants who reported that their arm felt more tight following the intervention also showed the least improvement on the F-M assessment and reported temporary discomfort following some of the training sessions. Not only does this demonstrate an alignment between participant's awareness of changes in their arm function and clinical outcome measures but it may also provide some insight into why these participants showed smaller improvements in the F-M measure compared to other participants. It must be noted that adverse effects were monitored throughout the intervention and adjustments to training were made. In the case of these two participants, discomfort was probably related to muscle fatigue and 
resolved following rest from training. However, this highlights the importance of constant monitoring and adaption of training schedules to reduce fatigue and increase the effectiveness of the treatment. Moreover, this information allowed us to gain insight into individual differences in changes in outcome measures that previously may have remained unexplained. Furthermore, our results highlight how participant perspectives can be used alongside traditional clinical assessments to provide greater insight into reported effectiveness. We have demonstrated that SAIL reduced impairments and that participants were aware of this. However, the system needs further development to achieve more effective, functional improvements.

The participant perspective data will also be very useful with respect to the development of this system. For example, it is clear that participants want a system that focuses on training distal upper limb joints as well as more proximal joints and could be used in a homesetting. In addition, the perspective data indicated that the next system should have a more varied task set to facilitate the enjoyment and motivation of some participants.

It is important to note that there are limitations to this study that may affect the generalizability of the findings. Foremost, as this was a feasibility study, the sample size used was small and may be underpowered $(\mathrm{N}=5$; although findings were consistent with previous work [18]). The question set that was used to gain participants' perspectives was designed in-house specifically for the purposes of evaluating and developing our ILC systems and was not a validated questionnaire. In addition, the lack of a control group within a pre-post assessment study design makes it difficult to determine whether the improvements observed are due to the intervention per se or are due to the fact that participants were doing some upper limb exercise. It would also be prudent to assess the effects of this rehabilitation system over time to see whether the improvements found remained. Thus, future work needs to trial this technology with a larger sample size and with post-intervention follow ups over a 3-6 month time period.

In evaluating and thinking about future development of the SAIL system through the participant perspective data, six broad issues that may be important for researchers to consider in the development of novel rehabilitation technologies and therapies were highlighted. It must be noted that these key points are, by themselves, not new concepts; however, they are often neglected in the design of technology-led rehabilitation systems [13]. Furthermore, there is much interaction between these concepts, and as such they should be considered holistically. Each of these six points will now be discussed in turn.

\section{A. Effectiveness: Specify what "Improvement" Means for your Study}

As mentioned above and consistent with previous research, participants in the current study clearly had different perceptions of the term improvement $[10,12]$. Despite participants showing reductions in motor impairments on the F-M, participants' views regarding the effectiveness of the treatment were mixed, with many indicating the desire for improvements on meaningful, everyday tasks. This demonstrates the importance of considering individual differences in rehabilitation goals and of specifying realistic levels of improvement at the start of any new study or treatment $[13,15]$. However, perceived recovery by patients who had survived stroke has been found to be strongly associated with hope [9]. Thus, although it is important set attainable goals, these goals should still present a challenge for the patient to achieve. It is worth noting that the types of improvement that this training may have provided were discussed at the beginning of the study with each participant; however, it would seem that this needs to be re-addressed in future studies.

\section{B. Train the Whole Upper Limb}

All participants in the current study reported a desire to also train the wrist and hand. Systematic reviews show that most rehabilitation robot trials have focussed on proximal upper limb movement [11, 23, 24] with consequent improvement limited to those joints. The need for more distal training is well documented in the literature [11, 18, 23, 24], although there are very few distal rehabilitation robotic systems available [11, 2326]. Furthermore, many current systems employing ES to assist wrist and hand movement use simplistic controllers which cannot provide precise movement and hence limit effectiveness [27]. Consequently, there is a tangible need in this area for model-based ES controllers, such as ILC $[19,20,21]$. To address the issue of non-significant functional improvements, future studies should train both distal and proximal muscles in the upper limb [23] and the tasks undertaken should focus on everyday ADLs that are important to the user $[9,12,13]$. 


\section{Flexibility of Rehabilitation Systems for Progression and Motivation}

Rehabilitation platforms must be flexible to allow tailoring at an individual level. For example, in the current study some participants felt that more varied tasks would have added to their motivation during training, whereas others felt the system was fine as it was. Participants need a range of options available to allow for task progression in terms of speed, range, complexity and dexterity, as well as adequate feedback to maintain motivation and appraise performance [9, 12, 13]. In addition, more patient-centred outcome measures are being developed and used to determine the types of task trained (e.g. MAM-16, [10]; COPM, [14]). This demands flexible systems that meet individual and changing needs, so that motivation and adherence to therapy are maximised. This may also assist in managing expectations of the therapy. Furthermore, future systems must be easily adjusted to individual participants, quick and easy to set-up and ideally usable in non-clinical settings, such as the home.

\section{Need for Portable, Home-Based Systems}

As many current robotic systems are designed for clinic-based use (see [23]; for review) the burdens of travel and time reported in the current study are as likely as with any intensive rehabilitation programme at external clinics. This is likely to affect adherence, motivation, satisfaction and consequently outcome. Indeed it was the participant who lived closest to the University in the current study that wished to have more training sessions; all of the other participants felt 18 was enough of a commitment (despite having felt that his would not be a problem at the start of the intervention).

Technological advances will encourage the design of home use rehabilitation systems. Systematic reviews of the emerging research in telerehabilitation for example, suggest positive outcomes with respect to clinical outcome measures, attendance and compliance by both patients and therapists [28, 29]. As well as alleviating time and travel commitments, home-based systems could reduce clinical costs, an important consideration in current economic climates and provide greater opportunity for more and self-directed practice. However, without therapists to oversee training, homebased systems will require special consideration with respect to the ease at which assistive devices can be set-up, task progression, and monitoring facilities (to ensure that participants are using the devices appropriately) [13]. In addition, long-term clinical and cost-effectiveness of such systems is required [28, 29].

\section{E. Avoiding Discomfort Due to Over-Use}

Many people experience some form of temporary discomfort following ES and robotic therapy, with muscle aches consistent with using a device intensively. Discomfort caused by rehabilitation exercises can reduce the likelihood of the person training [9]. In the current study, the therapist was always on hand to support, monitor and adapt the training schedules of the two participants who experienced temporary discomfort during training. The extent of education given to the participant and carers regarding possible discomfort should be considered, particularly in home-based systems when over-use may result in adverse reactions.

\section{F. The Value of Participant Perspectives}

Participant involvement is essential in ensuring that rehabilitation technologies have tangible impact for participants. Feedback from participants is useful for evaluating systems, influencing the evolution of individual prototype systems, such as SAIL, but more importantly in telling us where systems should be going in the future. In addition, it is becoming increasingly popular to use outcome measures that are meaningful and important to the participant and that measure global aspects of performance and participation [10, 11]. The feasibility and relevance of participants' desires should be critically considered, but lessons learnt from participant feedback can be applied to develop and improve novel rehabilitation systems. With this in mind, in the case of SAIL, the next step is to develop the system in a clinical based environment so that ES can be applied to facilitate movement of the whole upper limb, including the hand and wrist. Work is currently underway to achieve this. Once clinical feasibility is established for the new system, we will investigate piloting it within a home based environment, as this would address issues with regards to travel and time involved in rehabilitation training. The current study also highlighted that asking participant perspectives provided evidence that the participants themselves observed changes in the motor impairments of their impaired arm, such as they were more aware of the arm and that it felt more apart of them again, that would not have been captured if only standardised clinical assessments of motor function had been used. 
In conclusion, participant feedback was used to evaluate a novel rehabilitation platform combining robot-aided therapy, ES and ILC technology. Importantly the participant perspectives were in line with the clinical data demonstrating small but significant reductions in upper limb impairment that were reported to be of benefit by participants. Both clinical findings and participant feedback have indicated what further developments are needed to achieve more effective, functional improvement. Participants' perspectives also provided more in depth insight into perceptions of effectiveness and usability, highlighting six important and integrated points that researchers developing new rehabilitation technologies, especially those for the upper limb may wish to consider. Thus, the consideration and application of participants' perspectives provided informative feedback with regards to the feasibility and effectiveness of this rehabilitation platform.

\section{ACKNOWLEDGEMENTS}

This work was supported by the Engineering and Physical Sciences Research Council. Grant No. EP/G014078/1.

\section{REFERENCES}

[1] Stroke association. Stroke statistics, 1-7, 2006

[2] Carroll K, Murad S, Eliahoo J, Majeed A. Stroke incidence and risk factors in a population -based prospective cohort study. Health Statistics Quarterly 2001; 12: 18-26.

[3] Barreca S, Wolf SL, Fasoli S, Bohannon R. Treatment interventions for the paretic upper limb of stroke survivors: $A$ critical review. Neurorehabil Neural Repair 2003; 17: 220-6. http://dx.doi.org/10.1177/0888439003259415

[4] De Kroon JR, IJzerman MJ, Chae J, Lankhorst GJ, Zilvold G. Relation between stimulation characteristics and clinical outcome of the upper extremity in stroke. Rehabil Med 2005; 37: 65-74.

\section{http://dx.doi.org/10.1080/16501970410024190}

[5] Langhorne $\mathrm{P}$, Coupar F, Pollock A, et al. Motor recovery after stroke: a systematic review. Lancet Neurol 2009; 8: 741-51. http://dx.doi.org/10.1016/S1474-4422(09)70150-4

[6] Fugl-Meyer AR, Jääskö L, Leyman I, Olsson S, Steglind S. The post-stroke hemiplegic patient. 1. A method for evaluation of physical performance. Scand $\mathrm{J}$ Rehabil Med 1975; 7: 13-31.

[7] Lyle RC. A performance for assessment of upper limb function in physical rehabilitation treatment and research. Int J Rehabil Res 1981; 4: 483-92. http://dx.doi.org/10.1097/00004356-198112000-00001

Burridge JH, Haugland M, Larsen B, et al. Patients' perception of the benefits and problems of using the actigait implanted drop-foot stimulator. J Rehabil Med 2008; 40: 8735.

http://dx.doi.org/10.2340/16501977-0268

[9] Barker RN, Gill TJ, Brauer SG. Factors contributing to upper limb recovery after stroke: A survey of stroke survivors in Queensland Australia. Disabil Rehabil 2007; 29: 981-9. http://dx.doi.org/10.1080/09638280500243570
[10] Chen CC, Granger CV, Peimer CA, Moy OJ, Wald S. Manual Ability Measure (MAM-16): A preliminary report on a new patient-centred and task oriented outcome measure of hand function. J Hand Surg 2005; 30: 207-16.

http://dx.doi.org/10.1016/j.jhsb.2004.12.005

[11] Timmermans AAA, Seelen HAM, Geers RPJ, et al. Sensorbased arm skill training in chronic stroke patients: Results on treatment outcome, patient motivation, and system usability. IEEE Trans Neural Syst Rehabil Eng 2010; 18: 284-92. http://dx.doi.org/10.1109/TNSRE.2010.2047608

[12] Donovan-Hall MK, Burridge J, Dibb B, Ellis-Hill C, Rushton D. The views of people with spinal cord injury about the use of functional electrical stimulation. Artificial Organs 2011; 35: 204-11. http://dx.doi.org/10.1111/j.1525-1594.2011.01211.x

[13] Hochstenbach-Waelen A, Seelen HAM. Embracing change: practical and theoretical considerations for successful implementation of technology assisting upper limb training in stroke. J Neuroeng Rehabil 2012; 9: 52. http://dx.doi.org/10.1186/1743-0003-9-52

[14] Law M, Baptiste S, Carswell-Opzoomer A, McColl MA Polatajko H, Pollock N, Canadian Occupational Performance Measure. Toronto, ON: CAOT Publications ACE, 1991.

[15] Clark MS, Smith DS. Factors contributing to patient satisfaction with rehabilitation following stroke. Int J Rehabil Res 1998; 21: 143-53. http://dx.doi.org/10.1097/00004356-199806000-00003

[16] Lewis GN, Woods C, Rosie JA, McPherson KM. Virtual reality games for rehabilitation: Perspectives from the users and new directions, In International Conference on Virtual Rehabilitation (ICVR), Zurich, Switzerland; 2011 June.

[17] Hughes AM, Freeman CT, Burridge JH, Chappell PH, Lewin $\mathrm{PL}$, Rogers E. Feasibility of iterative learning control mediated by functional electrical stimulation for reaching after stroke. Neurorehabil Neural Repair 2009; 23: 559-68.

http://dx.doi.org/10.1177/1545968308328718

[18] Hughes AM, Burridge JH, Freeman CT, et al. Stroke participants' perceptions of robotic and electrical stimulation therapy: a new approach. Disabil Rehabil: Assist Technol 2011; 6: 1308.

http://dx.doi.org/10.3109/17483107.2010.509882

[19] Freeman C, Tong D, Meadmore K, et al. Phase-lead Iterative Learning Control Algorithms for Functional Electrical Stimulation based Stroke Rehabilitation. Proceedings of the Institution of Mechanical Engineers - Part I: Journal of Systems \& Control Engineering 2011; 225: 850-9. http://dx.doi.org/10.1177/0959651811408976

[20] Freeman C, Rogers E, Hughes AM, Burridge JH, Meadmore $\mathrm{KL}$. Iterative Learning Control in Healthcare: Electrical Stimulation and Robotic-assisted Upper Limb Stroke Rehabilitation. IEEE Control Syst Mag 2012; 32: 18-43.

[21] Freeman CT, Hughes AM, Burridge JH, Chappell PH, Lewin PL. Rogers E. A model of the upper extremity using FES for stroke rehabilitation. J Biomech Eng 2009; 131: 031011-12. http://dx.doi.org/10.1115/1.3005332

[22] Meadmore KL, Hughes AM, Freeman CT, et al. Functiona Electrical Stimulation mediated by Iterative Learning Contro and 3D robotics reduces motor impairment in chronic stroke. J Neuroeng Rehabil 2012; 9: 32. http://dx.doi.org/10.1186/1743-0003-9-32

[23] Timmermans AAA, Seelen HAM, Willmann RD, Kingma $\mathrm{H}$ Technology-assisted training of arm-hand skills in stroke: concepts on reacquisition of motor control and therapist guidelines for rehabilitation technology design. J Neuroeng Rehabil 2009; 6: 1 .

[24] $\mathrm{Hu} X \mathrm{~L}$, Tong KY, Song R, et al. Quantitative evaluation of motor functional recovery process in chronic stroke patients during robot-assisted wrist training. J Electromyogr Kinesiol 19: $639-50$ 
[25] Knutson JS, Harley MY, Hisel TZ, Chae J. Improving hand function in stroke survivors: A pilot study of contralaterally controlled functional electrical stimulation in chronic hemiplegia. Arch Phys Med Rehabil 2007; 88: 513-20. http://dx.doi.org/10.1016/j.apmr.2007.01.003

[26] Buschfort R, Brocke J, He $\beta$ A, Werner C, Waldner A, Hesse S. Arm studio to intensify upper limb rehabilitation after stroke: concept, acceptance, utilization and preliminary clinical results. J Rehabil Med 2010; 42: 310-4. http://dx.doi.org/10.2340/16501977-0517
[27] Zhang D, Guan TH, Widjaja F, Ang WT. Functional electrical stimulation in rehabilitation engineering: a survey. In Proc. Int Conv Rehab Eng Assistive Tech 2007; 221-6.

[28] Johanson $T$, Wild $C$, Telerehabilitation in stroke care - A systematic review. Telemed Telecare 2011; 17: 1-6. http://dx.doi.org/10.1258/itt.2010.100105

[29] Kairy D, Lehoux P, Vincent C, Visintin M. A systematic review of clinical outcomes, clinical process, healthcare utilization and costs associated with telerehabilitation. Disabil Rehabil 2009; 31: 427-47. http://dx.doi.org/10.1080/09638280802062553

(C) 2013 Meadmore et al.; Licensee Synergy Publishers.

This is an open access article licensed under the terms of the Creative Commons Attribution Non-Commercial License (http://creativecommons.org/licenses/by-nc/3.0/) which permits unrestricted, non-commercial use, distribution and reproduction in any medium, provided the work is properly cited. 